\title{
Lack of Cardiopulmonary Resuscitation Knowledge among Young Medical Doctors: A Worldwide Issue
}

\author{
Enrico Baldi
}

\begin{abstract}
Keywords: Cardiopulmonary resuscitation, Medical education, Medical student. Indian Journal of Critical Care Medicine (2021): 10.5005/jp-journals-10071-23701
\end{abstract}

\section{Dear Editor,}

I read with great interest the paper from Vineeth Chandran and Abraham ${ }^{1}$ demonstrating that the basic life support (BLS) knowledge among young doctors in India is very low. These results are unfortunately perfectly in line with a survey we recently carried out, endorsed by the ERC Research NET, which involved over 1.000 medical students just before their graduation from 99 universities in 14 different European countries. ${ }^{2}$ Also in that survey, we pointed out that cardiac arrest and cardiopulmonary resuscitation (CPR) knowledge among medical students in the period leading up to their graduation is scarce throughout Europe, highlighting how this is probably a worldwide issue that can affect patients' care. The respondents of our and Vineeth Chandran's survey, indeed, are the doctors of the future. Moreover, considering that there are evidence highlighting that the BLS and advanced cardiac life support (ACLS) knowledge of doctors currently operating is suboptimal, if not poor, in different parts of the world, ${ }^{3,4}$ we can speculate that it is a long-standing problem. Interestingly, the life-saving technique knowledge among Vineeth Chandran's respondents was poor despite $96 \%$ of students had attended non-structured BLS classes in the past. This has also been found in our recent European survey, where it was highlighted that many courses did not adhere to current international guidelines and many students reported only didactic theory classes without any BLS skills practice. Moreover, we pointed out that many students who are taught CPR during their initial undergraduate years do not undergo any subsequent refresher training and this may have affected their long-term knowledge, stressing that, as also revealed by Vineeth Chandran, regular refresh is needed to maintain CPR competencies.

That is why, considering all these aspects, we have recently promoted a European Resuscitation Council guidance note regarding "Mandatory cardiopulmonary resuscitation competencies for undergraduate healthcare students in Europe". ${ }^{5}$ The guidance note proposes a stepwise approach starting with CPR training in the first year of education, with an annual refresh, up to advanced life support (ALS) training for senior undergraduate students. Moreover, instructing personnel of these courses needs to teach according to international guidelines and peer-teaching programs to facilitate these high-volume courses should also be considered. This teaching program should become part of the general curriculum of each healthcare educational institution to achieve the necessary goals for healthcare students: to understand and deliver lifesaving competencies to their patients, and others who collapse within their community.

Considering the evidence that is accumulating more and more from different countries of the world regarding the lack of awareness of life-saving techniques by healthcare students,
Cardiac Intensive Care Unit, Arrhythmia and Electrophysiology and Experimental Cardiology, Fondazione IRCCS Policlinico San Matteo, Pavia, Italy; Department of Molecular Medicine, Section of Cardiology, University of Pavia, Pavia, Italy

Corresponding Author: Enrico Baldi, Cardiac Intensive Care Unit, Arrhythmia and Electrophysiology and Experimental Cardiology, Fondazione IRCCS Policlinico San Matteo, Pavia, Italy; Department of Molecular Medicine, Section of Cardiology, University of Pavia, Pavia, Italy, Phone: +39 382 501276, e-mail: enrico.baldi@unipv.it

How to cite this article: Baldi E. Lack of Cardiopulmonary Resuscitation Knowledge among Young Medical Doctors: A Worldwide Issue. Indian J Crit Care Med 2021;25(1):106.

Source of support: EB salary is partially funded from the European Union's Horizon 2020 research and innovation program under acronym ESCAPE-NET, registered under grant agreement no 733381.

Conflict of interest: None

our stepwise approach to training these students in lifesaving maneuvers should be encouraged all around the world. This would allow the creation of future generations of healthcare professionals who have all the tools to ensure the best chance of survival for their patients who suffer a cardiac arrest.

\section{References}

1. Vineeth Chandran K, Abraham SV. Basic life support: need of the hour-a study on the knowledge of basic life support among young doctors in india. Indian J Crit Care Med 2020;24(5):332-335. DOI: 10.5005/jp-journals-10071-23442.

2. Baldi E, Contri E, Bailoni A, Rendic K, Turcan V, Donchev N, et al. Final-year medical students' knowledge of cardiac arrest and CPR: we must do more!. Int J Cardiol 2019;296:76-80. DOI: 10.1016/j. ijcard.2019.07.016.

3. Einav S, Wacht O, Kaufman N, Alkalay E. Cardiopulmonary arrest in primary care clinics: more holes than cheese: a survey of the knowledge and attitudes of primary care physicians regarding resuscitation. Isr J Health Policy Res 2017;6(1):22. DOI: 10.1186/s13584017-0148-1.

4. Roshana S, Batajoo KH, Piryani RM, Sharma MW. Basic life support: Knowledge and attitude of medical/paramedical professionals. World J Emerg Med 2012;3(2):141-145. DOI: 10.5847/wjem.j.i ssn.1920-8642.2012.02.011.

5. Baldi E, Savastano S, Contri E, Lockey A, Conaghan P, Hulme J, et al. Mandatory cardiopulmonary resuscitation competencies for undergraduate healthcare students in Europe. A European resuscitation council guidance note. Eur J Anaesthesiol 2020;37(10):13. DOI: 10.1097/EJA.0000000000001272. 